\title{
Nerve fibers that were not stained with the non-specific acetylcholinesterase (NsAchE) method, and TRPV1- and IB4-positive nerve fibers in the rat cornea
}

\author{
Hiroshi Nakagawa, ${ }^{1,4)}$, Akio Hiura ${ }^{2)}$, Masato Mitome ${ }^{3)}$, and Kazunori Ishimura ${ }^{4)}$ \\ ${ }^{1)}$ Pediatric Dentistry, Tokushima University Hospital, ${ }^{2}$ Department of Histology and Oral Histology, \\ Institute of Health Bioscience, University of Tokushima Graduate School, ${ }^{3}$ Department of Pediatric \\ Dentistry, Institute of Health Bioscience, University of Tokushima Graduate School and ${ }^{4}$ Department \\ of Anatomy and Cell Biology, Institute of Health Bioscience, University of Tokushima Graduate \\ School, Tokushima, Japan
}

\begin{abstract}
Previously, we noticed the presence of nerve fiber-like structures in a whole mount preparation of the rat cornea that had not been stained with the non-specific acetylcholinesterase (NsAchE) method. These nerve-like fibers were projected into the central area of the cornea, forming a mesh-like pattern. The aim of this study is to examine the properties of these mesh-like fibers using the following two methods : their sensitivity to capsaicin and the detection of isolectin B4 (IB4) - and capsaicin receptor TRPV1 (transient receptor potential vanilloid 1 ) -reactivities. The mean disappeared area of non-stained fibers after NsAchE treatment was $26 \%$ of the total areas in the neonatally capsaicin-treated cornea. Bunches composed of fine IB4-positive nerve fibers were seen in a whole mount preparation. There were connections between the bunches, producing a mesh-like pattern similar to that of the fibers that were not stained with NsAchE. Fine TRPV1-immunoreactive (ir) nerve fibers were also shown to form bunches, with connections between each bunch observed in whole mount preparations. Thus, TRPV1-ir nerve fibers seem to densely innervate the rat corneal subepithelial stroma and are distinct from the NsAchEpositive nerve fibers. The TRPV1-ir fine nerve fibers overlapped with the IB4-positive nerve fibers, suggesting that the mesh-like fibers that were not stained with NsAchE are fine nociceptive sensory nerve fibers because of their sensitivity to capsaicin and similar distribution pattern to IB4- and TRPV1-positive nerve fibers. J. Med. Invest. 56 : 157-165, August, 2009
\end{abstract}

Keywords : TRPV1, IB4, Nociceptive sensory nerve fibers, cornea, capsaicin

\section{INTRODUCTION}

The cornea is supplied by unmyelinated and fine

Received for publication June 26, 2009 ; accepted July 21, 2009.

Address correspondence and reprint requests to Hiroshi Nakagawa, Pediatric Dentistry, Tokushima University Hospital, Kuramoto-cho, Tokushima 770-8503, Japan and Fax : +81-88633-9132. myelinated trigeminal sensory nerve fibers (1). The density of innervation in the corneal epithelium is 300-600 times higher than that in the skin and 2040 times than that in the tooth pulp (2). Corneal sensory nerves subserve key protecting responses to various stimuli. Activation of the corneal nerves by noxious stimuli induces a predominant sensation of pain followed by escape behaviors. Desiccation of the corneal surface stimulates superficially located 
free nerve endings in the epithelium and initiates a "corneal reflex" with blinking, leading to the spread of a tear film over the eye, thereby rewetting the corneal surface.

Many staining techniques ; i.e., nonspecific acetylcholinesterase (NsAchE, 3-6), gold chloride ( $\mathrm{AuCl}$, $7,8)$ and double staining with $\mathrm{NsAchE}$ and $\mathrm{AuCl}$ (9) have been used to visualize the corneal nerve fibers. Furthermore, there have been numerous reports relating to the neurotransmitters of the corneal nerve, such as substance P (SP, 10-13), calcitonin gene-related peptide (CGRP, 14-18), and protein gene product (PGP, 13, 19), but no studies have tried to examine the distribution of transient receptor potential vanilloid 1 (TRPV1)-positive nerve fibers in the cornea.

TRPV1 is a receptor of capsaicin that is preferentially expressed in small sensory neurons and nerve fibers. Recently, Nakamura, et al . (20) reported that an average of TRPV1-labeled trigeminal neurons of the rat was $22.8 \%$ for the neurons innervating to the cornea. Besides, TRPV1 expression was demonstrated in the corneal epithelial cells of the mouse (21). These TRPV1-expressing corneal cells were considered to release inflammatory mediators (IL6 , IL-8) in response to noxious stimuli or infection (21).

As for the channel receptors, the transient receptor potential (TRP) are a superfamily of cation channels that display a great diversity of sensing mechanisms. They are widely distributed in mammalian tissues and respond to a wide range of environmental signals, including temperature, touch, pain, osmolarity, taste, protons (low $\mathrm{pH}$ ), and pheromones (22). Among them, the receptors of temperature are TRPV1-4, TRPM8, and TRPA1 (23-28). Electrophysiological data imply that specific corneal receptors are activated by thermal or mechanical stimulation or both (29-33) ; therefore, it stands to reason that the cornea would have receptors capable of detecting thermal and mechanical stimuli.

We found that the pattern and density of the subepithelial corneal nerve fibers stained by the NsAchE method did not differ significantly between the control and capsaicin-treated neonate rats (19) ; i.e., the NsAchE method used in our study did not stain capsaicin-sensitive corneal nerves. In addition, we noticed the presence of many nerve fiber-like structures that had not been stained with NsAchE in the rat cornea. These fibers ran towards the central area and formed a mesh-like architecture. Recently, Murata and Masuko have reported the presence of TRPV1-immunoreactive (ir) nerve fibers in the rat cornea (34). They demonstrated the existence of TRPV1-ir nerve fibers, but not their distribution. The purpose of the present study is to examine the properties of these mesh-like fibers. Since there is no direct method that can determine whether the mesh-like fibers are sensory nerve fibers, we indirectly investigated this matter using the following two parameters : their sensitivity to capsaicin, and the detection of isolectin B4 (IB4) - and TRPV1-reactivity. IB4 exclusively labels small-type neurons and unmyelinated sensory fibers. Thus, IB4-reactive neurons and fibers are almost all with TRPV1-reactive small neurons or unmyelinated sensory fibers.

\section{MATERIALS AND METHODS}

All the experiments followed the guidelines of the Ethics Committee of the International Association for the Study of Pain (35) and were approved by the Committee on Experimental Animals and the Ethical Treatment of Animals of the University of Tokushima.

Enucleations of the eye were always made under anesthesia with pentobarbital sodium.

\section{Influence of capsaicin on the mesh-like fibers that were not stained with the NsAchE method}

A single dose of capsaicin $(50 \mathrm{mg} / \mathrm{kg}$, Nacalai Tesque Inc., Kyoto, Japan) was injected into the dorsal skin (s. c) of rat pups $(n=4)$ on day 3 after birth. The same number of age-matched animals received only a vehicle (10\% ethanol and $10 \%$ Tween 80 in saline) and served as controls. The bilateral corneas with narrow scleral margins in each animal were excised from enucleated eyes at 15 days after capsaicintreatment. Briefly, after dipping the specimens in DPB (Dulbeco's phosphate buffered saline), the iris and ciliary body were removed. The specimens were fixed in a solution of $4 \%$ paraformaldehyde in $0.1 \mathrm{M}$ phosphate buffer (pH 7.4) overnight. Subsequently, they were stored in $0.1 \mathrm{M}$ phosphate buffered saline (PBS, pH 7.4) containing $10 \%$ sucrose for 1 day at $4^{\circ} \mathrm{C}$. The corneas were not stained at all. The corneas from capsaicin-treated animals were microscopically photographed at a magnification of $\times 20$. Entire corneal figures were made as montages at a final magnification of $\times 76$. The total area of the capsaicin-treated cornea containing the mesh-like fibers was measured by the NIH image processing 
and analysis program. The lost area of mesh-like fibers in the capsaicin-treated cornea was estimated from the total area.

\section{Detection of IB4 - and TRPVI-reactivities}

1) Paraffin-embedding of sections by the avidin biotin complex $(A B C)$ method to detect TRPVI and $P G P$

a) The sections from an entire eye embedded materials

Enucleated eyes were fixed in a solution of Bouin for 12-24 hours. Subsequently, they were washed in $70 \%$ ethanol for a week. The specimens were dehydrated in isopropanol, cleared in xylene, embedded in paraffin, and cut into slices of 5-7 $\mu \mathrm{m}$ thickness. The sections were deparaffinized in xylene, passed through a graded series of isopropanol (96\%-70\%), and placed in water. After the sections had been placed in hot water $\left(92^{\circ} \mathrm{C}, 15 \mathrm{~min}\right)$, they were dipped in PBS and preincubated with $5 \%$ bovine serum albumin (BSA)-PBS containing $1 \%$ Triton X-100 at room temperature (RT) for $30 \mathrm{~min}$, followed by incubation in $1 \%$ BSA containing $0.5 \%$ Triton X-100 (RT, $3 \times 5 \mathrm{~min}$ ). Then, the specimens were incubated in 1\% BSA-PBS containing 30\% avidin (RT, 20 $\mathrm{min}$ ), before being washed in 1\% BSA-PBS, incubated in 1\% BSA-PBS containing 30\% biotin (RT, 20 $\mathrm{min})$, and then washed again in 1\% BSA-PBS. They were reacted with rat polyclonal anti-TRPV1 (raised in rabbit, $1: 300,16^{\circ} \mathrm{C}$, overnight). After being rinsed in $0.5 \%$ BSA-PBS (RT, $3 \times 5 \mathrm{~min}$ ), they were incubated with anti-rabbit biotin-labeled secondary antibodies $\left(1: 200,37^{\circ} \mathrm{C}, 45 \mathrm{~min}\right)$. After being rinsed in $0.5 \%$ BSA-PBS, they were incubated in avidinbiotin-peroxidase dissolved in $1 \% \mathrm{BSA}-\mathrm{PBS}\left(37^{\circ} \mathrm{C}\right.$, $45 \mathrm{~min}$ ). The specimens were prereacted with $0.06 \%$ $\mathrm{Ni}^{2+}$-intensified diamino benzidine (DAB, $0.01 \%$ ) solution ( $5 \mathrm{~min}$ ) and reacted with the same solution containing $0.01 \% \mathrm{H}_{2} \mathrm{O}_{2}$ (8 min). They were then washed in water, dehydrated in isopropanol, and cleared in xylene. Finally, the sections were embedded in Depex. Similarly, immunostaining with protein gene product (PGP, $1: 1500$, Ultra Clone Ltd. Isle of Wight, England) as a marker of peripheral nerve fibers was also performed in paraffin sections using mouse polyclonal antibody.

b) Sections from the excised cornea

The corneas excised from the enucleated eyes were also fixed and paraffin embedded. The sections were reacted in the same way as described above.

\section{2) Staining of IB4 in the whole mount preparations (immunofluorescent method)}

The bilateral corneas with narrow scleral margins were excised from the enucleated eyes in each animal. Briefly, after dipping the specimens in DPB, the iris and ciliary body were removed. They were fixed in a solution of $4 \%$ paraformaldehyde with or without $0.2 \%$ picric acid in $0.1 \mathrm{M} \mathrm{PBS}\left(4^{\circ} \mathrm{C}\right.$, overnight). After fixation, they were stored in $0.1 \mathrm{M}$ PBS containing $10 \%$ sucrose $\left(24 \mathrm{~h}, 4^{\circ} \mathrm{C}\right)$. Then, the corneas were pre-incubated in 5\% BSA-PBS containing 1\% Triton X-100 (RT, 30 min). Subsequent rinsing in $1 \%$ BSA containing $0.5 \%$ Triton X-100 (RT, $2 \times 5$ $\mathrm{min}$ ), was followed by incubation of the corneas in FITC-coupled IB4 $(10 \mathrm{mg} / \mathrm{ml}$, Vector Lab. Inc. Burlingame, USA) for $24 \mathrm{~h}$ at $4^{\circ} \mathrm{C}$. After they had been rinsed in PBS (RT, $3 \times 5 \mathrm{~min}$ ), the specimens were embedded in $1 \%$ propyl gallate.

3) Double staining with IB4 and TRPVI of whole mount preparations (immunofluorescent method)

The excision and fixation of the corneas were performed using the same method as noted above. After incubation of the corneas in the FITC-coupled IB4 $(10 \mathrm{mg} / \mathrm{ml})$ for $24 \mathrm{~h}$ at $4^{\circ} \mathrm{C}$, they were washed in PBS, preincubated in 5\% BSA-PBS containing $1 \%$ Triton X-100 (RT, $30 \mathrm{~min}$ ), and rinsed in 1\% BSA containing $0.5 \%$ Triton X-100 (RT, $3 \times 5 \mathrm{~min}$ ). Then, the specimens were incubated for $24 \mathrm{~h}$ at $4^{\circ} \mathrm{C}$ in rat polyclonal anti-TRPV1 (raised in rabbit, $1: 2000$, Trans Genic Inc., Kumamoto, Japan). They were then washed in PBS (RT, $3 \times 5 \mathrm{~min}$ ) before being incubated with the secondary antibodies (Texas Red conjugated goat anti-rabbit IgG, $1: 400$, Rockland Inc. Gilbertsville, USA) for $2 \mathrm{~h}$ at $37^{\circ} \mathrm{C}$. The subsequent procedures were the same as mentioned above.

Specimens devoid of the primary antiserum and IB4 were examined as controls. Immunofluorescence materials were observed using a fluorescence microscope (DP70, Olympus, Tokyo, Japan).

\section{RESULTS}

\section{Sensitivity of the mesh-like fibers to capsaicin}

Fiber networks were clearly observed in the control corneas. They ran to the central area and formed a mesh-like structure (Fig. 1a, b). The mesh-like fibers markedly disappeared or were distorted in the capsaicin-treated corneas (Fig. 1c, d). The loss 

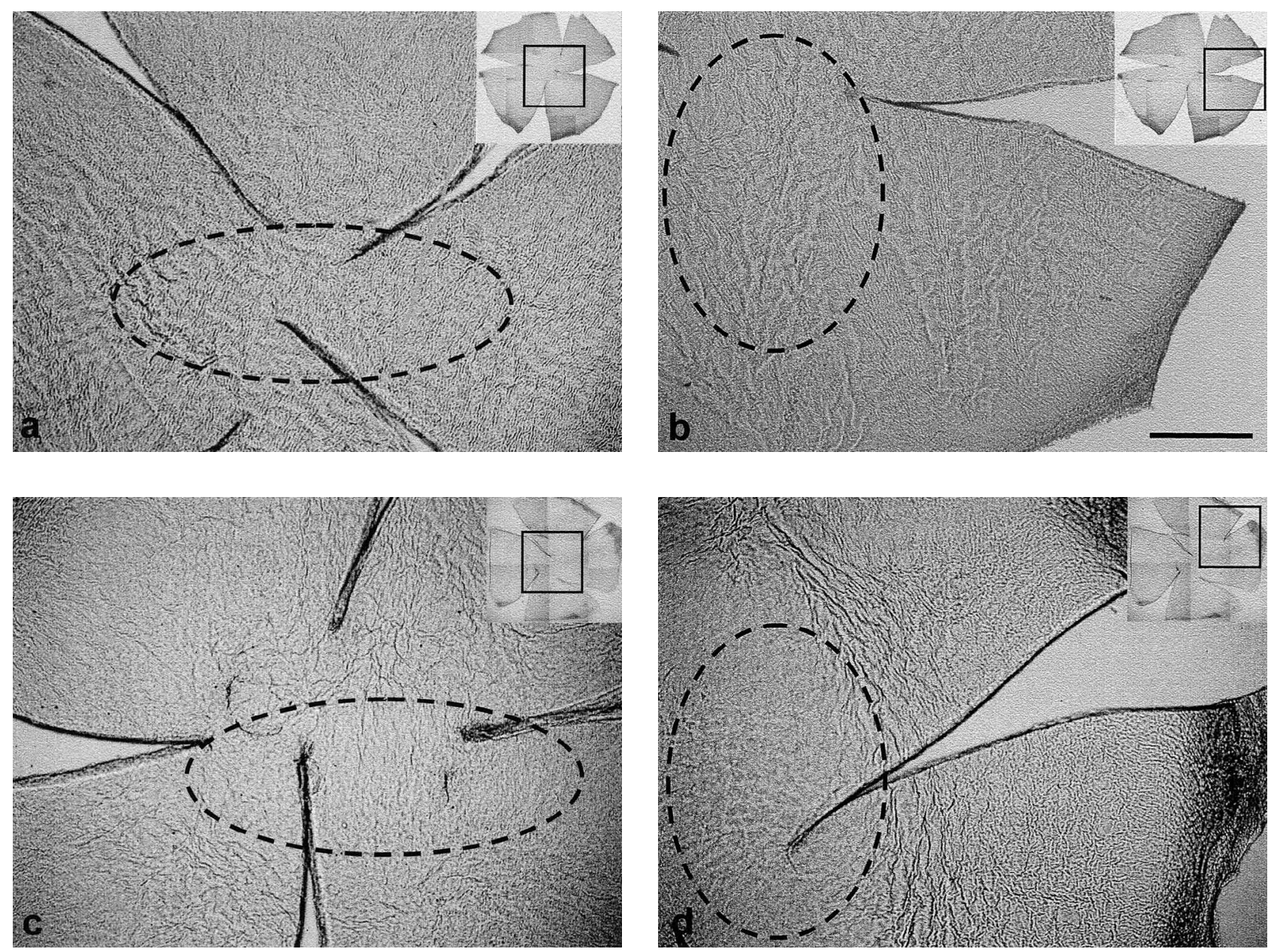

Fig. 1 Mesh-like fibers that were not stained with NsAchE in whole mount corneas 15 days after neonatal capsaicin- (50 mg/kg, c, d) or vehicle-treatment $(\mathrm{a}, \mathrm{b})$. The fiber network is clearly seen in the control corneas. They ran into the central area and formed mesh-like structures $(a, b)$. The mesh-like fibers markedly disappeared and were distorted in the capsaicin-treated cornea (c, d). Especially, the loss of fibers was conspicuous in the center of the cornea (dotted area). The clover leaf-like small inset figures show whole mount corneas that have been reduced in size. a- $d$ : Higher magnification of the square regions in the inset figures. Scale bar : $0.5 \mathrm{~mm}$.

of fibers and their distorted arrangement were conspicuous especially in the center of the cornea. A mean area of $26.2 \%$ of the total area of the capsaicintreated corneas showed a loss of the mesh-like fibers (Table 1). No disappearance or distortion of the mesh-like fibers was observed in the control corneas (Fig. 1).
TRPVI-immunoreactive (ir) and PGP-ir nerve fibers in the paraffin-embedded sections

A few TRPV1-ir fibers were seen in the base of the epithelium (Fig. 2a). However, many TRPV1-ir fibers were seen among the obliquely sectioned epithelium (Fig. 2b). No TRPV1-ir nerve fibers were

Table 1. The lost area of nerve fiber-like structure in the cornea 15 days after treatment with capsaicin.

\begin{tabular}{c|c|c|c}
\hline animal & area $\left(\mathrm{cm}^{2}\right)$ & lost $\left(\mathrm{cm}^{2}\right)$ & 21.0 \\
\hline A & 413.2 & 86.6 & 19.2 \\
\hline B & 721.8 & 138.9 & 37.3 \\
\hline C & 278.5 & 103.8 & 27.5 \\
\hline D & 463.5 & 127.3 & $26.2 \pm 8.2$ \\
\hline
\end{tabular}



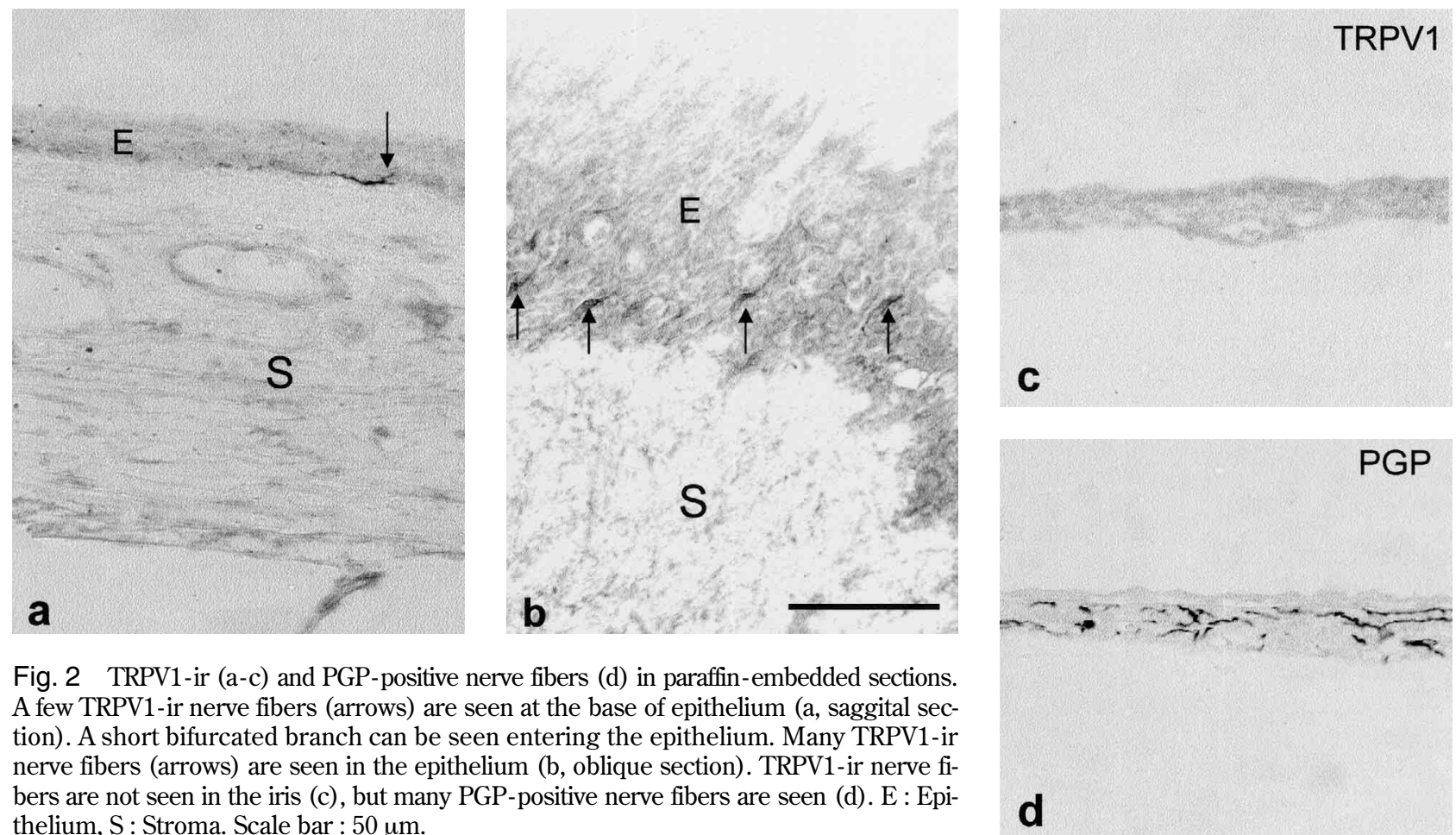

Fig. 2 TRPV1-ir (a-c) and PGP-positive nerve fibers (d) in paraffin-embedded sections. A few TRPV1-ir nerve fibers (arrows) are seen at the base of epithelium (a, saggital section). A short bifurcated branch can be seen entering the epithelium. Many TRPV1-ir nerve fibers (arrows) are seen in the epithelium (b, oblique section). TRPV1-ir nerve fibers are not seen in the iris (c), but many PGP-positive nerve fibers are seen (d). E : Epithelium, S : Stroma. Scale bar : $50 \mu \mathrm{m}$.

bunches consisting of fine IB4-positive nerve fibers and with connections between the bunches (Fig. 3 b-d). Thus, IB4-positive nerve fibers made a mesh like pattern similar to that of the fibers that were not stained with NsAchE (Fig. 3a). TRPV1-ir fine nerve fibers were also seen to form bunches (Fig. 4a). The
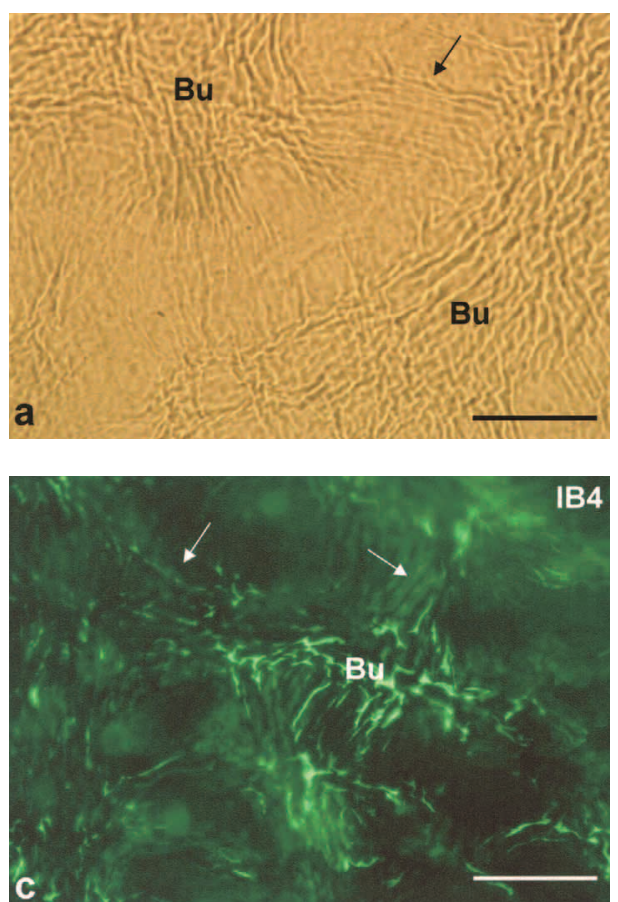
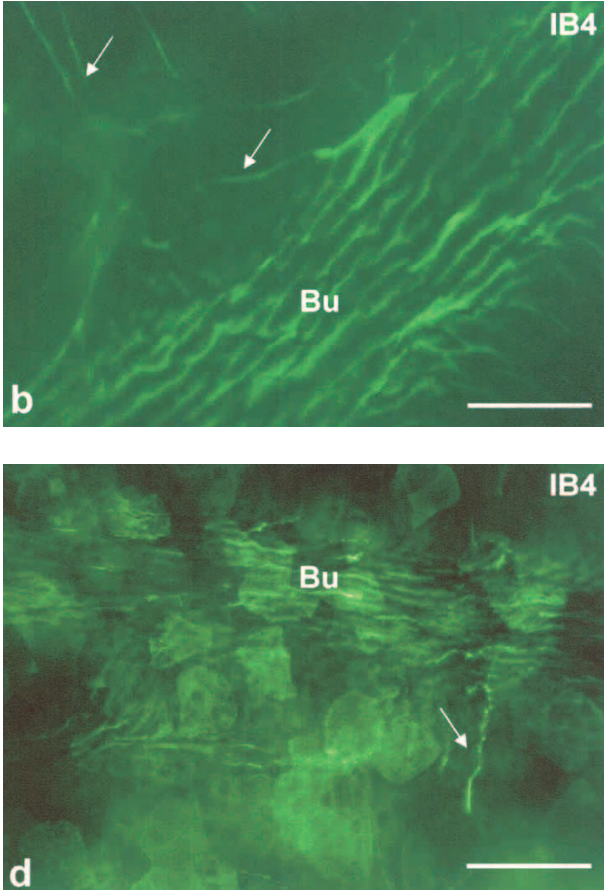

Fig. 3 IB4-positive nerve fibers in a whole mount preparation (b-d). A bunch (Bu) is composed of IB4-positive fine nerve fibers. The connections between the bunches are indicated by arrows. The distribution of IB4-positive nerve fibers (b) is seen as a mesh-like pattern similar to that of the non-stained fibers observed by the use of an orange fluorescent filter (a). Scale bars : a. c. d : $50 \mu \mathrm{m}, \mathrm{b}: 25 \mu \mathrm{m}$. 

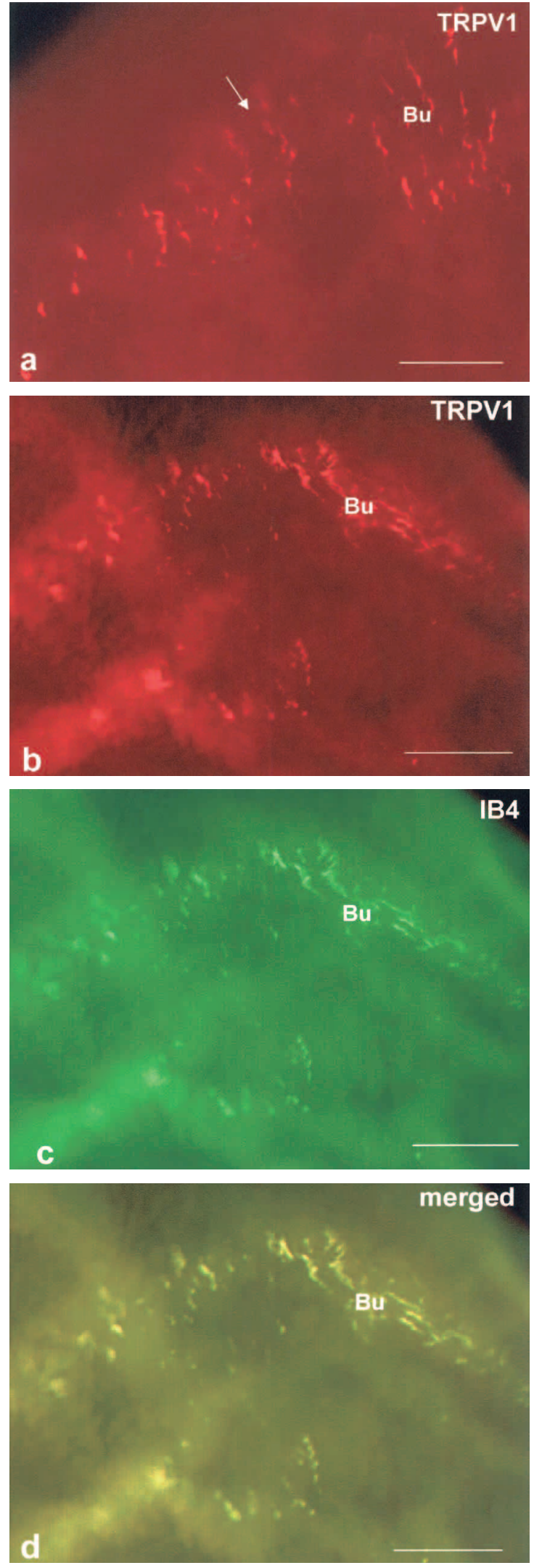

Fig. 4 TRPV1-ir fine nerve fibers $(a, b)$ in whole mount preparations. The bunches $(\mathrm{Bu})$ are connected to each other by TRPV1ir fine nerve fibers (a, arrow). The bunch-forming nerve fibers show waves due to their rise and fall in the stroma (b). Note that TRPV1-ir nerve fibers make mesh-like structures similar to IB4positive nerve fibers (c). A merged figure (d) shows the overlapping of IB4 and TRPV1 in the nerve fibers (d). Scale bars : a : 25 $\mu \mathrm{m}, \mathrm{b}-\mathrm{d}: 50 \mu \mathrm{m}$. bunches were connected to each other by TRPV1ir positive fine nerve fibers and made a mesh-like structure similar to the IB4-positive nerve fibers (Fig. 4a). The merged figures of the IB4-positive nerve fibers and TRPV1-ir positive nerve fibers show that they overlap (Fig. 4b, c, d).

\section{DISCUSSION}

Recently, we demonstrated the pattern and density of the corneal subepithelial nerve fibers stained by the NsAchE method (19). No significant difference in nerve fiber density was found between NsAchE-positive nerve fibers in the control and capsaicin-treated corneas (19). Accordingly, the NsAchE-positive nerve fibers are capsaicin-resistant. Simultaneously, we noticed that there were many mesh-like fibers in the corneas that were not stained with NsAchE. The mesh-like fibers were not thought to be collagen fibers due to their branching and connections. Whether or not the mesh-like fibers are nerve fibers was examined in this study using the following parameters : their sensitivity to capsaicin and reactivity to TRPV1-antibody and plant lectin IB4.

\section{The sensitivity of the mesh-like fibers to capsaicin}

Many fiber-like structures were seen in the nonstained control corneas. The fibers ran from the periphery to the central area, forming mesh-like patterns. These mesh-like fibers were severely diminished in the capsaicin-treated corneas. The disappearance and distortion of the fibers was marked in the center of the cornea. The mean loss of meshlike fiber area was $26 \%$ of the total area in the capsaicin-treated corneas. These results indicate that the fibers that were not stained with NsAchE are capsaicin-sensitive. The number of PGP-ir nerve fibers in the cornea 15 days after capsaicin injection at two days of age was decreased compared to that in the control cornea (19). Furthermore, corneal lesions were induced by neonatal capsaicin application in proportion to the dose of drug and survival time after treatment (36). The first occurrence of a lesion at the center of cornea (36) was closely correlated with the loss of mesh-like fibers in the present capsaicin-treated corneas. Thus, the mesh-like fibers that were not stained with NsAchE are sensitive to capsaicin ; and therefore, seem to be nociceptive sensory nerve fibers. 


\section{TRPV1-immunoreactivity in corneal nerves}

Murata and Masuko (34) reported the presence of TRPV1-ir nerve fibers in the rat cornea. They demonstrated the existence of TRPV1, but not the pattern or distribution of TRPV1-ir nerve fibers in the cornea. The present study showed that a few TRPV1-ir nerve fibers were present in saggital sections of the cornea. However, many TRPV1-ir nerve fibers were seen in oblique sections of the cornea. TRPV1-ir fibers were not seen in the iris, although many PGP-ir fibers were present in this tissue. Since the PGP-ir fibers in the iris may be autonomic nerves, the absence of TRPV1-ir fibers in the iris acts sure as a negative control for TRPV1-ir nerve fibers.

TRPV1-ir fine nerve fibers formed bunches in the whole mount preparations. The bunches were connected to each other by thin filament bundles ; therefore, TRPV1-ir nerve fibers also form a meshlike pattern similar to the fibers that were not stained with NsAchE. The results suggest that a considerable number of TRPV1-positive nerve fibers are present in the rat cornea.

\section{IB4-reactivity in corneal nerves}

IB4 is a plant lectin extracted from the seeds of Griffonia (Bandeiraea) simplicifolia. The first characterization of small sensory neurons in the dorsal root ganglion (DRG) and TG of the rat was made using IB4 conjugated to horseradish peroxidase (HRP) by Streit, et al . (37). IB4 exclusively labeled small-type neurons smaller than $800 \mu \mathrm{m}^{2}$, unmyelinated sensory fibers, and their central terminals in the substantia gelatinosa (SG) of the spinal dorsal horn. Silverman and Kruger (16) reported that fine-diameter IB4-positive nerve fibers were seen throughout the corneal epithelium and stroma, ramifying extensively, radiating both horizontally and vertically, and making a mesh-like pattern. In the present study, bunches of IB4-positive nerve fibers showed connections with each other and made mesh-like patterns similar to those seen in the aforementioned study (16) and those of fibers that were not stained with NsAchE. This suggests that the mesh-like fibers that were not stained with NsAchE are the same as the IB4-positive nerve fibers.

Coexistence of IB4 and TRPVI-ir in corneal nerve fibers

TRPV1-ir fine nerve fibers formed bunches and a mesh-like distribution pattern similar to IB4-positive nerve fibers. TRPV1- and IB4-reactivity nearly perfectly overlapped in the merged figures. This indicates that TRPV1 colocalizes with IB4-positive nerve fibers in the cornea. Guo, et al. (38) showed that more than $50 \%$ of small- to medium-sized L5 DRG and TG neurons in rats express TRPV1 (neuron size : 200-1100 $\mu \mathrm{m}^{2}$, peak $400-800 \mu \mathrm{m}^{2}$ ). TRPV1 colocalized with IB4 in these neurons (78\% TRPV1= IB4+, 67\% IB4+=TRPV1). The overlapping of TRPV1 and IB4 in the corneal nerves may reflect their coexistence in smaller TG neurons.

As a result, IB4-positive small neurons were found to be capsaicin sensitive and to play important roles in responses to TRPV1 agonists, heat (42$52^{\circ} \mathrm{C}$ ), and endogenous cannabinoids (39). Instillation of capsaicin $(30 \mu \mathrm{M}$ : mouse, $100 \mu \mathrm{M}$ : rat) on the cornea induced eye wiping a mean number of 20 (mouse) and 5 (rat) times in normal animals ; whereas, neonatally capsaicin-treated animals did so a mean of only 2.5 (mouse) and 0.9 (rat) times $(19,40)$. Capsaicin is a TRPV1 receptor agonist, which binds to the channel and induces chemical nociception. Therefore, the reduction of wiping at least in rats was considered to be closely related to a decrease in the number of capsaicin-sensitive, fibers that were not stained with NsAchE in the present study and PGP-ir nerve fibers (19). Together, TRPV1-ir and IB4-positive nerve fibers may have a major role in nociceptive sensation in the cornea.

\section{CONCLUSIONS}

1) The nerve fibers that were not stained with NsAchE are sensitive to capsaicin.

2) Many IB4-positive fine nerve fibers are present in the rat cornea. They formed a mesh-like pattern similar to the fibers that were not stained with NsAchE.

3) A considerable number of TRPV1-ir nerve fibers innervate the rat cornea. They also form a meshlike pattern similar to the IB4-positive nerve fibers.

The present results strongly suggest that the mesh-like fibers seen under non-stained conditions are capsaicin-sensitive and make bunches of fine IB4- or TRPV1-positive nerve fibers, indicating that they are nociceptive sensory nerve fibers.

The greater part of this study was presented at the "Workshop of Pain" held by the National Institute for Physiological Sciences on 22-23 January, 2009, at Okazaki, Japan. 


\section{REFERENCES}

1. Zander E, Weddell $\mathrm{G}$ : Observations on the innervation of the cornea. J Anat 85 : 68-93, 1951

2. Rozsa AJ, Beuerman RW : Density and organization of free nerve endings in the corneal epithelium of the rabbit. Pain $14: 105-120,1982$

3. Robertson DM, Winkelmann RK : A wholemount cholinesterase technique for demonstrating corneal nerves : observations in the albino rabbit. Invest Ophthal $9: 710-715,1970$

4. Tervo $\mathrm{T}:$ Histochemical demonstration of cholinesterase activity in the cornea of the rat and the effect of various denervations on the corneal nerves. Histochemistry $47: 133-143$, 1976

5. Tervo T, Palkama A : Innervation of the rabbit cornea. A histochemical and electron-microscopic study. Acta Anat 102 : 164-175, 1978

6. Ishida N, Cerro MD, Rao GN, Mathe M : Corneal stromal innervation. A quantitative analysis of distribution. Ophtalmic Res 16 : 139-144, 1984

7. Silverberg KR, Ogilvy CS, Borges LF : A modified gold chloride technique for optimal impregnation of nerves within corneal whole mounts and dura of the albino rat. J Histochem Cytochem 37 : 269-271, 1989

8. Jacot JL, Glover JP, Robinson WG Jr : Improved gold chloride procedure for nerve staining in whole mounts of rat corneas. Biotech Histochem 70 : 277-284, 1995

9. Jacot JL, Glover JP, Robinson WG Jr : Computer analysis of corneal innervation density using a novel double stain in rat corneal whole mounts. J Anat 191 : 191-199, 1997

10. Miller A, Costa M, Furness JB, Chubb IW : Substance $\mathrm{P}$ immunoreactive sensory nerves supply the rat iris and cornea. Neurosci Lett 29 : 243-249, 1981

11. Shimizu Y : Localization of neuropeptides in the cornea and uvea of the rat : an immunohistochemical study. Cell Mol Biol 28 : 103-110, 1982

12. Tervo K, Tervo L, Eranko L, Eranko O : Substance $\mathrm{P}$ immunoreactive nerves in the rodent cornea. Neurosci Lett 25 : 95-97, 1981

13. Marfurt CF, Kingsley RE, Echtenkamp SE: Sensory and sympathetic innervation of the mammalian cornea. Invest Ophthalmol Vis Sci 30 : 461-472, 1989

14. Jones MA, Marfurt CF : Calcitonin gene-related peptide and corneal innervation : a developmental study in the rat. J Comp Neurol 313 : 132150, 1991

15. Colin S, Kruger L : Peptidergic nociceptive axon visualization in whole-mount preparations of cornea and tympanic membrane in rat. Brain Res 398 : 199-203, 1986

16. Silverman JD, Kruger L : Lectin and neuropeptide labeling of separate populations of dorsal root ganglion neurons and associated "nocieptor" thin axons in rat testis and cornea wholemount preparations. Somatosens Res 5 : 259267, 1988

17. Stone RA, Kuwayama Y, Terenghi G, Polak $\mathrm{JM}$ : Calcitonin gene-related peptide : occurrence in corneal sensory nerves. Exp Eye Res $43: 279-283,1986$

18. Stindl G, Skofitsh G : Immunohistochemical localization of calcitonin gene-related peptide in the rat eye. Response to capsaicin pretreatment. Ann NY Acad Sci 657 : 464-465, 1992

19. Hiura A, Nakagawa $H$ : Capsaicin-resistant, nonspecific acetylcholinesterase (NsAchE) reactive nerve fibers in the rat cornea: A quantitative and developmental study. Okajimas Folia Anat Jpn 81 : 75-84, 2004

20. Nakamura A, Hayakawa T, Kuwahara S, Maeda S, Tanaka K, Seki M, Mimura O : Morphological and immunohistochemical characterization of the trigeminal ganglion neurons innervating the cornea and upper eyelid of the rat. J Chem Neuroanat 34 : 95-101, 2007

21. Zhang F, Yang H, Wang Z, Mergler S, Liu H, Kawakita T, Tachado SD, Pan Z, Capo-Aponte JE, Pleyer U, Koziel H, Kao WWY, Reinach PS : Transient receptor potential vanilloid 1 activation induces inflammatory cytokine release in corneal epithelium through MAPK signaling. J Cell Physiol 213 : 730-739, 2007

22. Venkatachalam K, Montell C : TRP channels. Annu Rev Biochem 76 : 387-417, 2007

23. Tominaga $M$, Caterina $M J$, Malmberg $A B$, Rosen TA, Gilbert H, Skinner K, Raumann BE, Basbaum AI, Julius D : The cloned capsaicin receptor integrates multiple pain-producing stimuli. Neuron 21 : 531-543, 1998

24. Caterina MJ, Rosen TA, Tominaga M, Brake AJ, Julius D : A capsaicin receptor homologue with a high threshold for noxious heat. Nature 398 : 436-441, 1999

25. Smith GD, Gunthorpe MJ, Kelsell RE, Hayes PD, Reilly P, Facer P, Wright JE, Jerman JC, 
Walhin J-P, Ooi L, Egerton J, Charles KJ, Smart D, Randall AD, Anand P, Davis JB : TRPV3 is a temperature-sensitive vanilloid receptor-like protein. Nature 418 : 186-190, 2002

26. Peier AM, Moqrich A, Hergarden AC, Reeve AJ, Andersson DA, Story GM, Earley TJ, Dragoni I, McIntyre P, Bevan S, Patapoutian A : A TRP channel that senses cold stimuli and menthol. Cell 108 : 705-715, 2002

27. Story GM, Peier AM, Reeve AJ, Eid SR, Mosbacher J, Hricik TR, Earley TJ, Hergarden AC, Andersson DA, Hwang SW, McIntyre P, Jegra T, Bevan S, Patapoutian A : ANKTM1, a TRP-like channel expressed in nociceptive neurons, is activated by cold temperature. Cell $112: 819-829,2003$

28. Madrid R, Rodriguez TD, Meseguer V, Acosta MC, Belmonte C, Viana F : Contribution of TRPM8 channels to cold transduction in primary sensory neurons and peripheral nerve terminals. J Neurosci 26 : 12512-12525, 2006

29. Belmonte C, Giraldez F : Responses of cat corneal sensory receptors to mechanical and thermal stimulation. J Physiol 321 : 355-368, 1981

30. Giraldez F, Grijo E, Belmonte C : Response characteristics of corneal sensory fibers to mechanical and thermal stimulation. Brain Res 177 : 571-576, 1979

31. Acosta MC, Tan ME, Belmonte C, Gallar J : Sensations evoked by selective mechanical, chemical, and thermal stimulation of the conjunctiva and cornea. IOVS 42 : 2063-2067, 2001

32. Beuerman RW, Tanelian DL: Corneal pain evoked by thermal stimulation. Pain $7: 1-14$, 1979

33. Kenshalo DR : Comparison of thermal sensitivity of the forehead, lip, conjunctiva and cornea. J Appl Physiol 15 : 987-991, 1960

34. Murata Y, Masuko S : Peripheral and central distribution of TRPV1, substance P and CGRP of rat corneal neurons. Brain Res 1085 : 87-94, 2006

35. Zimmermann $\mathrm{M}:$ Ethical guidelines for investigations of experimental pain in conscious animals. Pain 16 : 109-110, 1983

36. Hiura A, Nakagawa $\mathrm{H}$ : Induction of corneal lesion and nerve fiber sprouting by neonatal capsaicin application depends on the dose of the drug and survival time after treatment. Okajimas Folia Anat Jpn $82:$ 57-66, 2005

37. Streit EJ, Schulte BA, Balentine JD, Spicer SS : Histochemical localization of galactosecontaining glycoconjugates in sensory neurons and their processes in the central and peripheral nervous system of the rat. J Histochem Cytochem $33:$ 1042-1052, 1985

38. Guo A, Vulchanova L, Wang J, Li X, Elde R: Immunocytochemical localization of the vanilloid receptor 1 (VR1) : Relationship to neuropeptides, the P2X3 purinoceptor and IB4 binding sites. Eur J Neurosci 11 : 946-958, 1999

39. Liu M, Willmott NJ, Michael GJ, Priestrey $\mathrm{JV}$ : Differential $\mathrm{pH}$ and capsaicin responses of Griffonia simplicifolia IB4 (IB4)-positive and IB4-negative small sensory neurons. Neuroscience $127: 659-672,2004$

40. Hiura A, Ishizuka H : Quantitative electronmicroscopic analyses of pulpal nerve fibres in the mouse lower incisor after neonatal capsaicin treatment. Arch Oral Biol 57 : 1085-1090, 1992 